\title{
NOTE \\ Virology \\ Distribution and phylogenetic analysis of Dabieshan tick virus in ticks collected from Zhoushan, China
}

\author{
Changqiang $\mathrm{ZHU}^{1)}$, Ting $\mathrm{HE}^{2)}$, Ting $\mathrm{WU}^{3)}$, Lele $\mathrm{Al}^{1)}$, Dan $\mathrm{HU}^{1}{ }^{1)}$, Xiaohong YANG ${ }^{1)}$, \\ Ruichen LV $^{1}$, Lu YANG $^{1}$, Heng LV $^{11}$ and Weilong $\operatorname{TAN}^{1}{ }^{1 *}$ \\ 1)Eastern Theater Command Centers for Disease Control and Prevention, Nanjing, 210002, China \\ ${ }^{2)}$ Children's Hospital of Nanjing Medical University, Nanjing, 210002, China \\ 3) Jinling Hospital Nanjing, Nanjing, 210002, China
}

J. Vet. Med. Sci.

82(8): 1226-1230, 2020

doi: 10.1292/jvms.20-0081

Received: 16 February 2020

Accepted: 28 May 2020

Advanced Epub:

10 June 2020
ABSTRACT. Dabieshan tick virus (DBV) belongs to Phlebovirus and its pathogenicity to human and animals is unknown. To investigate the presence of Dabieshan tick virus in Zhoushan, 353 ticks were collected from May 2018 to October 2019. The detection result showed that the average prevalence rate among these samples was $30.3 \%$ (107 positives out of 353 samples), which means DBVs are widely distributed in tick populations in Zhoushan of China. In a phylogenetic analysis based on the nucleotide sequences of the $L$ and S segments of the virus (ZS-DBS-2018 tick virus) in the study, it clustered with Dabieshan tick virus (KM817666.1, KM817733.1) with a $97.1 \%$ and $99.6 \%$ nucleotide identity, respectively. Further studies involving virus isolation are required to characterize Dabieshan tick virus and to expand the geographical distribution of the sampled ticks.

KEY WORDS: Dabieshan tick virus, phlebovirus, tick, Zhoushan

Ticks are important vectors for the transmission of pathogens including viruses with significant human and animal health impact [3]. The genus Phlebovirus including a large group of virus members are associated with ticks, which were usually named tickborne phleboviruses (TBPVs), such as severe fever with thrombocytopenia syndrome virus (SFTSV), Heartland virus (HRTV), Hunter Island group virus (HIGV), Lihan tick virus, Yongjia tick virus 1 and Dabieshan tick virus $[1,4,6,8,11,13]$.

In recent years, more and more attention has been paid to newly emerging TBPVs, which can induce serious human diseases. From 2010 to 2016, there had been more than 10,000 SFTSV infected cases distributed in 23 provinces in China, with the average mortality of 5.3\% [14]. Dabieshan tick virus is one of the novel pathogenic TBPVs and was first identified in China in 2015 [6]. So far, no infectious cases in humans have been reported to be associated with Dabieshan tick virus. However, it is very important to further study the isolation and epidemiological knowledge of Dabieshan tick virus.

From May 2018 to October 2019, tick sampling was carried out at 2 locations in Zhoushan (Fig. 1). At each site, the drag-flag method was performed to collect questing ticks and ticks infesting domesticated animals, mainly cattle, goats and dogs were collected using ophthalmic forceps at animal shelters. Each tick specimen was individually kept alive in a separate tube, transferred to the laboratory and identified morphologically using appropriate taxonomic criteria, and nested PCR assay objective to the tick 16S ribosomal RNA were conducted for the final identification $[2,7,10]$. All ticks were stored at $-80^{\circ} \mathrm{C}$ until further testing.

Ticks were thawed and homogenized using a glass grinder. The homogenates were centrifuged at $12,000 \mathrm{~g}$ for $10 \mathrm{~min}$ at $4^{\circ} \mathrm{C}$ but only the supernatant was kept. The supernatant of each sample was filtered through $0.22 \mu \mathrm{m}$ Pellicon II filters (Millipore, Billerica, MA, USA). The viral RNA was extracted with Viral RNA Mini Kit (QIAGEN, Hilden, Germany) following the manufacturer's recommendations (QIAamp Viral RNA Mini Handbook Download: https://www.qiagen.com/cn/resources/ resourcedetail? $\mathrm{id}=\mathrm{c} 80685 \mathrm{c} 0-4103-49$ ea-aa72-8989420e3018\&lang=en), RNA was eluted in $30 \mu l$ RNase-free $\mathrm{H}_{2} \mathrm{O}$ and stored at $-80^{\circ} \mathrm{C}$. Reverse transcription was carried out using the 1 st cDNA synthesis kit (TaKaRa, Kusatsu, Japan), according to the manufacturer's protocol.

For Dabieshan tick virus of phlebovirus detection, the L segment of virus was amplified by PCR using the DBSF-01 and DBSR1,100 primers. Samples positive for the Dabieshan tick virus were also amplified for the full sequence amplification of L segment and $\mathrm{S}$ segment of the virus by PCR. The L and S segment primers, were designed according to the highly homologous gene sequences of Dabieshan (KM817733) tick virus using the Primer Premier 5 (Premier Biosoft, San Francisco, CA, USA). All PCR

*Correspondence to: Tan, W.: njcdc@163.com

(Supplementary material: refer to PMC https://www.ncbi.nlm.nih.gov/pmc/journals/2350/)

(C2020 The Japanese Society of Veterinary Science

This is an open-access article distributed under the terms of the Creative Commons Attribution Non-Commercial No Derivatives (by-nc-nd) License. (CC-BY-NC-ND 4.0: https://creativecommons.org/licenses/by-nc-nd/4.0/) 


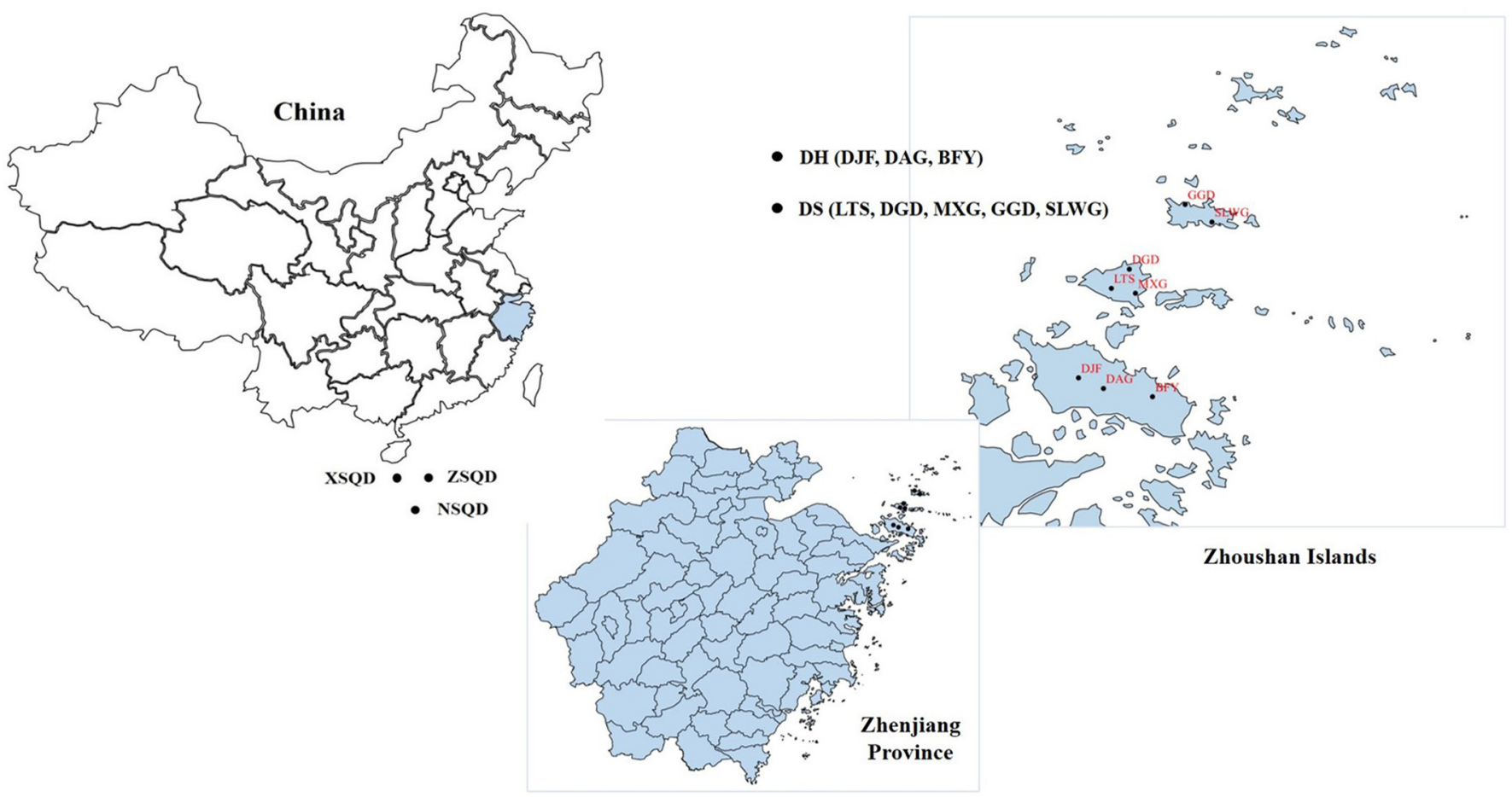

Fig. 1. Sampling locations of ticks in in Zhoushan of China. XSQD, Xishaqundao; ZSQD, Zhongshaqundao; NSQD, Nanshaqundao; DH, Dinghai; DJF, Dajianfeng; DAG, Daangang; YJV, Bianfuyan; DS, Daishan; LTS, Laitoushan; DGD, Dagangdun; MXG, Moxingang; GGD, Gaogangdun; SLWG, Shuangluanwanggang.

Table 1. Primer sequences used in this study

\begin{tabular}{cllc}
\hline Targets & \multicolumn{1}{c}{ Primer names } & \multicolumn{1}{c}{ Sequences (5'-3') } & Product sizes (bp) \\
\hline Dabieshan virus L segment & DBSF-01 & GCCAGCACTAGTTCCCTAG & 1,100 \\
& DBSR-1100 & GCCCTCCGTAGTTGATGGCG & \\
& DBSF-802 & CTAGAGAAGCCAGATCTTA & 1,188 \\
& DBSR-1990 & GGCTCATCTCCCTCTTCTAC & \\
& DBSF-1805 & CTTCCACAAGTCGGGGAACT & 1,295 \\
& DBSR-3100 & TGGCTCTGAGGATGAAGGC & \\
& DBSF-2877 & GACCATAATGAAGCAGGTTTG & 1,323 \\
& DBSR-4200 & CTGTGGTGGCCCTTTCGTAA & \\
& DBSF-4015 & GAAAAGGAGGTGGCCAAAGAAG & 1,785 \\
& DBSR-5800 & CTGTGGGTTCAGTCTTCT & \multirow{2}{*}{1,190} \\
& DBSF-5299 & CATGTTGGAATGGGGCC & \\
& DBSR-end & GGAGCTGGTTCAATCTCTC & 1,005 \\
\hline Dabieshan virus S segment & DBS-SF-01 & CACACAAAGACCCCTACCTT & \\
& DBS-SR-1005 & CCACCCCCAGCTTCTTG & 929 \\
& DBS-SF-826 & GAGCAGGACACCCAGGACA & \\
& DBS-SR-1755 & CACAAAGACCCCCTACC & \\
\hline
\end{tabular}

reactions $\left(30 \mathrm{cycles}\right.$ of $98^{\circ} \mathrm{C}$ for $10 \mathrm{sec}, 65^{\circ} \mathrm{C}$ for $15 \mathrm{sec}$, and $68^{\circ} \mathrm{C}$ for $1 \mathrm{~min}$ ) were performed using $50 \mu l$ reaction mix containing $2 \mu l$ extracted cDNA, $10 \mu l$ PCR Buffer, 20 pmol of each primer, $4 \mu l$ dNTP $(2.5 \mathrm{mM})$ and $1 \mu l$ Taq DNA polymerase (PrimeSTAR GXL, TaKaRa). The primers used for PCR and subsequent nucleotide sequencing are listed in Table 1.

The PCR products were purified using the QIAquick Gel Extraction kit (QIAGEN, Venlo, Netherlands) and sequenced in both directions by an ABI 3730 DNA Analyzer (Invitrogen, Beijing, China). Viral nucleotide and deduced amino acid sequences were analyzed and assembled by the DNASTAR software package (Lasergene). Complete or partial nucleotide sequences of the L and S segments of phlebovirus were obtained from GenBank. MEGA7.0 software was used to construct the maximum-likelihood evolutionary tree [5].

In present study, a total of 353 ticks were collected. Of these, 138 samples belonged to the genus Rhipicephalus haemaphysaloides, and 215 ticks were identified as Haemaphysalis longicornis (Table 2). All samples were tested for Dabieshan 
Table 2. Collected tick species and positive rates for Dabieshan tick virus in Zhoushan, China

\begin{tabular}{|c|c|c|c|c|c|c|c|c|c|c|c|}
\hline \multirow{3}{*}{ Strain } & \multicolumn{4}{|c|}{ DH (DJF, DAG, BFY) } & \multicolumn{4}{|c|}{ DS (LTS, DGD, MXG, GGD, SLWG) } & \multirow{3}{*}{$\begin{array}{c}\text { Total } \\
\text { (unfed ticks) }\end{array}$} & \multirow{3}{*}{$\begin{array}{c}\text { Total } \\
\text { (fed ticks) }\end{array}$} & \multirow{3}{*}{ Total } \\
\hline & \multicolumn{2}{|c|}{$\begin{array}{c}\text { Rhipicephalus } \\
\text { haemaphysaloides }\end{array}$} & \multicolumn{2}{|c|}{$\begin{array}{c}\text { Haemaphysalis } \\
\text { longicornis }\end{array}$} & \multicolumn{2}{|c|}{ R. haemaphysaloides } & \multicolumn{2}{|c|}{ H. longicornis } & & & \\
\hline & Unfed ticks & Fed ticks & Unfed ticks & Fed ticks & Unfed ticks & $\overline{\text { Fed ticks }}$ & Unfed ticks & Fed ticks & & & \\
\hline $\begin{array}{l}\text { Dabieshan } \\
\text { tick virus }\end{array}$ & $1 / 16$ & $9 / 40$ & $4 / 23$ & $23 / 57$ & $5 / 17$ & $16 / 65$ & $5 / 36$ & $44 / 99$ & $15 / 92$ & $92 / 261$ & $107 / 353$ \\
\hline
\end{tabular}

DH, Dinghai; DJF, Dajianfeng; DAG, Daangang; YJV, Bianfuyan; DS, Daishan; LTS, Laitoushan; DGD, Dagangdun; MXG, Moxingang; GGD, Gaogangdun; SLWG, Shuangluanwanggang.

Table 3. Pairwise comparison (\%) of nucleotide identity (upper diagonal) and amino acid identity (lower diagonal) for the $\mathrm{L}$ segment of Dabieshan tick virus in the study

\begin{tabular}{lcccccc}
\hline \multicolumn{1}{c}{ Strains } & 1 & 2 & 3 & 4 & 5 & 6 \\
\hline ZS-DBS-2018 (MN723842) & & 97.1 & 73.2 & 71.9 & 64.4 & 44.0 \\
DBV (KM817666) & 96.2 & & 73.8 & 74.2 & 64.9 & 44.7 \\
OKV (LC259521) & 79.1 & 79.9 & & 74.5 & 75.8 & 44.6 \\
LVV (KX452150) & 78.0 & 84.0 & 77.7 & & 72.9 & 45.2 \\
YJV (KM817704) & 65.0 & 65.7 & 84.2 & 78.0 & & 44.2 \\
SFTSV (KC505135) & 30.5 & 29.6 & 32.4 & 32.5 & 29.2 & \\
\hline
\end{tabular}

DBV, Dabieshan tick virus; LVV, Lesvos virus; SFTSV, Severe fever with thrombocytopenia syndrome virus; OKV, Okutama tick virus; YJV, Yongjia tick virus; ZS-DBS-2018, Dabieshan tick virus ZS-DBS-2018.

Table 4. Pairwise comparison (\%) of nucleotide identity (upper diagonal) and amino acid identity (lower diagonal) for the S segment of Dabieshan tick virus in the study

\begin{tabular}{lccccc}
\hline \multicolumn{1}{c}{ Strains } & 1 & 2 & 3 & 4 & 5 \\
\hline ZS-DBS2018 (MN723843) & & 99.6 & 67.6 & 61.3 & 50.4 \\
DBV (KM817733) & 100 & & 68.3 & 50.4 & 50.8 \\
OKV (LC259522) & 59.7 & 59.7 & & 77.2 & 53.6 \\
YJV (KM817764) & 54.9 & 54.9 & 71.1 & & 49.7 \\
AMD (KM 589348) & 29.3 & 29.3 & 36.5 & 29.8 & \\
\hline
\end{tabular}

AMD, American dog tick virus; DBV, Dabieshan tick virus; OKV, Okutama tick virus; YJV, Yongjia tick virus; ZS-DBS-2018, Dabieshan tick virus ZS-DBS-2018.

tick virus (Supplementary Fig. 1).

A 1,100 bp of the L segment gene of Dabieshan tick virus was successfully amplified from 107 out of 353 tick samples (30.3\%). Dabieshan tick virus identified from tick species Haemaphysalis longicornis in the Daishan (designated ZS-DBS-2018 tick virus) was selected for further full-length genome sequencing (Table 1). The 6,398 bp L segment and 1,010 bp S segment gene of ZS-DBS-2018 tick virus were obtained. The nucleotide sequence of this $6,398 \mathrm{bp}$ fragment showed $97.1 \%$ identity with that of Dabieshan tick virus (KM817666), 73.2\% with that of Okutamatick virus (LC259521), and 71.9\% with that of Lesvos virus (KX452150) (Table 3). The virus detected using phlebovirus genetic analysis in this study is hereafter referred to as Dabieshan tick virus. When compared with reference viruses (Yongjia, SFTS, and Bhanja viruses), the nucleotide sequence identity varied from 44.0 to $71.9 \%$. The nucleotide sequence of the 1,010 bp S segment gene also showed 99.6 and $67.6 \%$ identity with the Dabieshan tick virus and Okutama tick virus, respectively (Table 4). The nucleotide sequences of ZS-DBS-2018 tick virus are deposited in GenBank under the accession numbers MN723842 and MN723843.

In order to explore the phylogenetic origin of ZS-DBS-2018 tick virus, the large-scale maximum likelihood (ML) phylogeny trees were constructed based on the $6,398 \mathrm{bp} \mathrm{L}$ segment sequence and 1,010 bp S segment gene of ZS-DBS-2018 tick virus, and the ML tree showed that ZS-DBS-2018 tick virus clustered with the Dabieshan tick virus, the Yongjia tick virus, and Okutama tick virus and was most closely related with Dabieshan tick virus, a previously reported sequence isolated from $H$. hystricis in China [6, 9] (Figs. 2 and 3). At the nucleotide level, the L/S segment of ZS-DBS-2018 tick virus shared 97.1 and $99.6 \%$ identities with the Dabieshan tick virus, respectively. The ZS-DBS-2018 tick virus was most distantly related to other viruses in the same cluster. The human health burdens posed by the Dabieshan, Yongjia tick viruses as well as Okutama tick virus remain to be elucidated.

Therefore, for further studies of Dabieshan tick virus, we will expand the geographical distribution of the sampled ticks, and isolate the viruses through Vero-E6 cell culture and new-borne mice inoculation [12]. The study of Dabieshan tick virus in Zhoushan may contribute to the classification of phleboviruses and elucidate the evolutionary relationships among phleboviruses. 


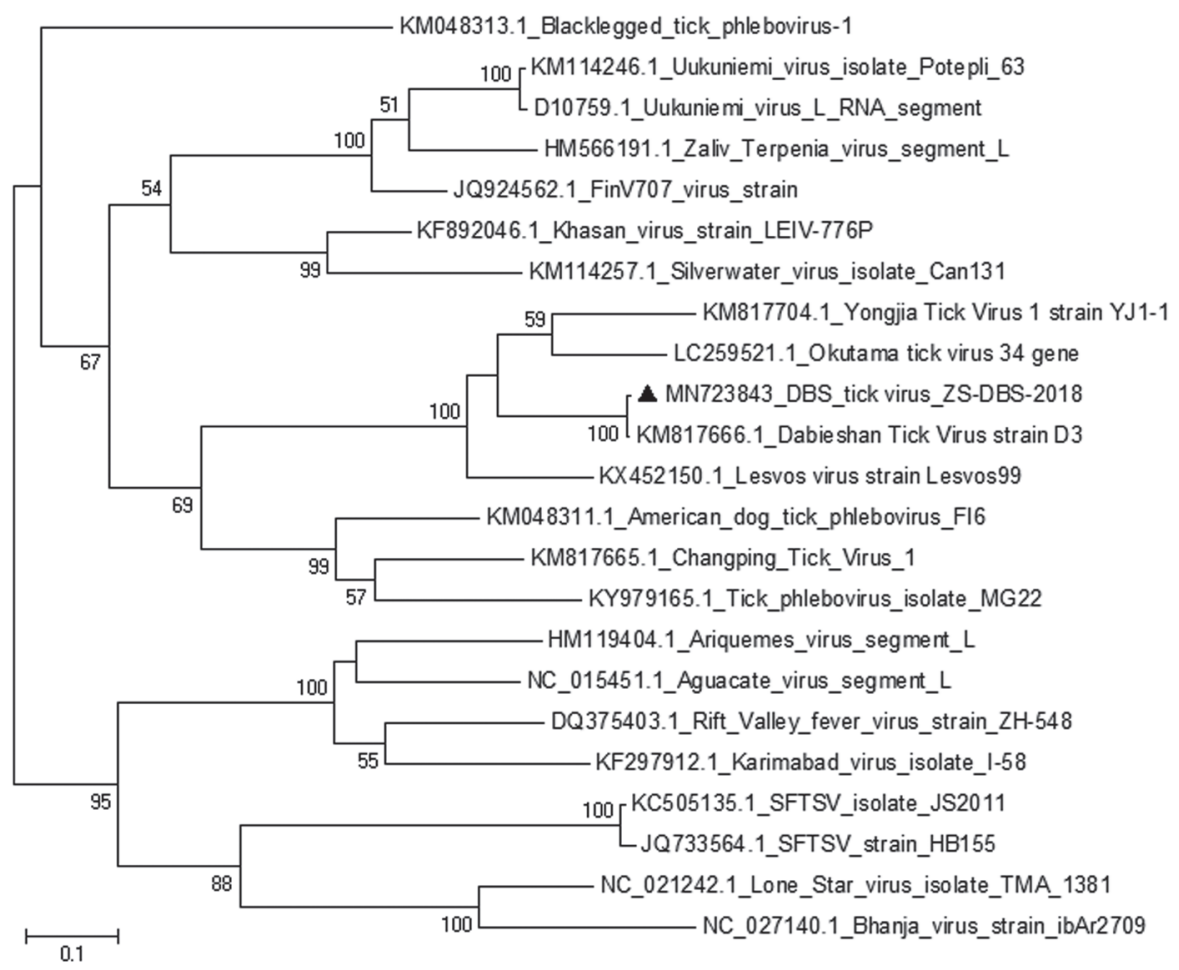

Fig. 2. Maximum likelihood phylogenetic trees based on a 6,398 bp nucleotide sequence of the $\mathrm{L}$ segment nucleotide sequence. The tests of nucleotide sequences based on the Tamura-Nei model. The numbers at the nodes represent bootstrap values of 1,000 replicates. Bootstrap probabilities above $50 \%$ are indicated near the branches. Sequences in the trees are indicated as GenBank accession number and strain name. Sequences of the present study are shown in bold. The triangles in the phylogenetic trees denote sequences derived in the study.

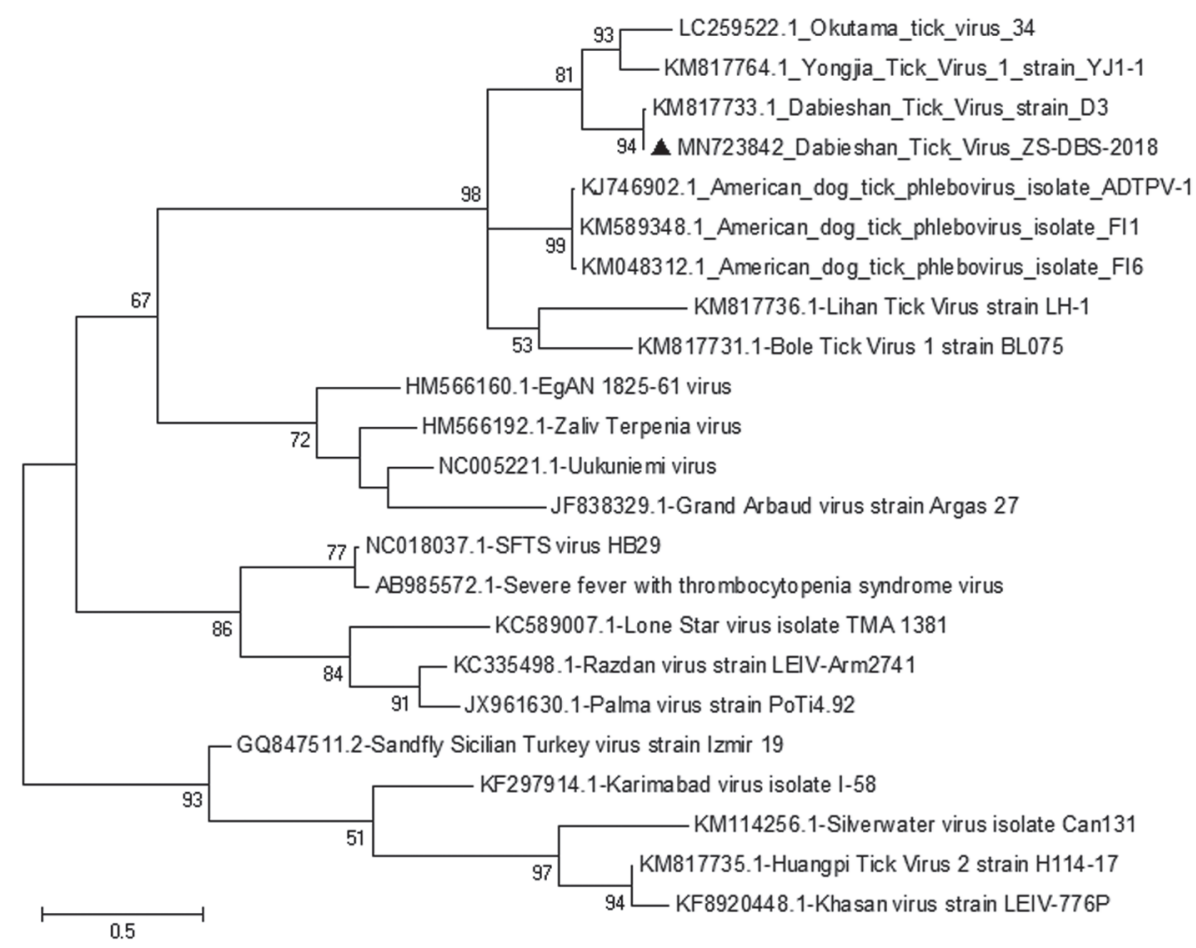

Fig. 3. Maximum likelihood phylogenetic trees based on a $1,010 \mathrm{bp}$ nucleotide sequence of the $\mathrm{S}$ segment nucleotide sequence. The tests of nucleotide sequences based on the Tamura-Nei model. The numbers at the nodes represent bootstrap values of 1,000 replicates. Bootstrap probabilities above $50 \%$ are indicated near the branches. Sequences in the trees are indicated as GenBank accession number and strain name. Sequences of the present study are shown in bold. The triangles in the phylogenetic trees denote sequences derived in the study. 
ACKNOWLEDGMENTS. This study was funded by grants from Project supported by National Natural Science Foundation of China (U1602223), General project of Jiangsu Health Committee (H2019015),Biosafety special project (17SAZ01), the National Major Infectious Diseases (2017ZX10303401-007),"333” project of Jiangsu Province (BRA 2018412).

\section{REFERENCES}

1. Bosco-Lauth, A. M., Panella, N. A., Root, J. J., Gidlewski, T., Lash, R. R., Harmon, J. R., Burkhalter, K. L., Godsey, M. S., Savage, H. M., Nicholson, W. L., Komar, N. and Brault, A. C. 2015. Serological investigation of heartland virus (Bunyaviridae: Phlebovirus) exposure in wild and domestic animals adjacent to human case sites in Missouri 2012-2013. Am. J. Trop. Med. Hyg. 92: 1163-1167. [Medline] [CrossRef]

2. Chitimia, L., Lin, R. Q., Cosoroaba, I., Braila, P., Song, H. Q. and Zhu, X. Q. 2009. Molecular characterization of hard and soft ticks from Romania by sequences of the internal transcribed spacers of ribosomal DNA. Parasitol. Res. 105: 907-911. [Medline] [CrossRef]

3. Dantas-Torres, F., Chomel, B. B. and Otranto, D. 2012. Ticks and tick-borne diseases: a One Health perspective. Trends Parasitol. 28: 437-446. [Medline] [CrossRef]

4. Fill, M. A., Compton, M. L., McDonald, E. C., Moncayo, A. C., Dunn, J. R., Schaffner, W., Bhatnagar, J., Zaki, S. R., Jones, T. F. and Shieh, W. J. 2017. Novel clinical and pathologic findings in a heartland virusassociated death. Clin. Infect. Dis. 64: 510-512. [Medline]

5. Hu, D., Zhu, C., Ai, L., He, T., Wang, Y., Ye, F., Yang, L., Ding, C., Zhu, X., Lv, R., Zhu, J., Hassan, B., Feng, Y., Tan, W. and Wang, C. 2018. Genomic characterization and infectivity of a novel SARS-like coronavirus in Chinese bats. Emerg. Microbes Infect. 7: 154. [Medline] [CrossRef]

6. Li, C. X., Shi, M., Tian, J. H., Lin, X. D., Kang, Y. J., Chen, L. J., Qin, X. C., Xu, J., Holmes, E. C. and Zhang, Y. Z. 2015. Unprecedented genomic diversity of RNA viruses in arthropods reveals the ancestry of negative-sense RNA viruses. eLife 4: 1-26. [Medline] [CrossRef]

7. Lu, X., Lin, X. D., Wang, J. B., Qin, X. C., Tian, J. H., Guo, W. P., Fan, F. N., Shao, R., Xu, J. and Zhang, Y. Z. 2013. Molecular survey of hard ticks in endemic areas of tick-borne diseases in China. Ticks Tick Borne Dis. 4: 288-296. [Medline] [CrossRef]

8. Matsumoto, N., Masuoka, H., Hirayama, K., Yamada, A. and Hotta, K. 2018. Detection and phylogenetic analysis of phlebovirus, including severe fever with thrombocytopenia syndrome virus, in ticks collected from Tokyo, Japan. J. Vet. Med. Sci. 80: 638-641. [Medline] [CrossRef]

9. Papa, A., Kontana, A., Tsioka, K., Saratsis, A. and Sotiraki, S. 2017. Novel phlebovirus detected in Haemaphysalis parva ticks in a Greek island. Ticks Tick Borne Dis. 8: 157-160. [Medline] [CrossRef]

10. Qin, X. C., Shi, M., Tian, J. H., Lin, X. D., Gao, D. Y., He, J. R., Wang, J. B., Li, C. X., Kang, Y. J., Yu, B., Zhou, D. J., Xu, J., Plyusnin, A., Holmes, E. C. and Zhang, Y. Z. 2014. A tick-borne segmented RNA virus contains genome segments derived from unsegmented viral ancestors. Proc. Natl. Acad. Sci. USA 111: 6744-6749. [Medline] [CrossRef]

11. Shi, J., Hu, Z., Deng, F. and Shen, S. 2018. Tick-borne viruses. Virol. Sin. 33: 21-43. [Medline] [CrossRef]

12. Xu, F., Yao, P. P., Zhu, H. P. and Xie, R. H. 2009. Molecular cloning and nucleotide sequence analysis of the S-segment in Hantavirus isolated in Zhejiang province. Chin. J. Zoonoses 25: 665-668.

13. Yoo, J. R., Heo, S. T., Park, D., Kim, H., Fukuma, A., Fukushi, S., Shimojima, M. and Lee, K. H. 2016. Family cluster analysis of severe fever with thrombocytopenia syndrome virus infection in Korea. Am. J. Trop. Med. Hyg. 95: 1351-1357. [Medline] [CrossRef]

14. Zhan, J., Wang, Q., Cheng, J., Hu, B., Li, J., Zhan, F., Song, Y. and Guo, D. 2017. Current status of severe fever with thrombocytopenia syndrome in China. Virol. Sin. 32: 51-62. [Medline] [CrossRef] 